\title{
Distribución vertical de la macroinfauna asociada a bivalvos en una planicie intermareal sedimentaria del sur de Chile
}

\author{
Vertical distribution of the macroinfauna associated to bivalves in a \\ sedimentary intertidal flat of southern Chile

\section{Karin Acuña ${ }^{1}$, Cristian Duarte ${ }^{2}$, Eduardo Jaramillo ${ }^{3}$, Heraldo Contreras ${ }^{4}$, Mario Manzano ${ }^{5}$ y Jorge M. Navarro ${ }^{1}$}

\author{
${ }^{1}$ Instituto de Ciencias Marinas y Limnológicas, Universidad Austral de Chile, Valdivia, Chile \\ ${ }^{2}$ Departamento de Ecología y Biodiversidad, Facultad de Ecología y Recursos Naturales, Universidad Andres Bello, Santiago, \\ Chile. cristian.duarte@unab.cl \\ ${ }^{3}$ Instituto de Ciencias de la Tierra y Evolución, Universidad Austral de Chile, Valdivia, Chile \\ ${ }^{4}$ Instituto de Fomento Pesquero, Centro Maricultura, Putemún, Chile \\ ${ }^{5}$ Ministerio de Obras Públicas, Valdivia, Chile
}

\begin{abstract}
The community structure of the macroinfauna inhabiting intertidal flats, as well as its distribution inside the sediment column, can be influenced by a combination of physical and biological factors. The objective of this study was to evaluate the vertical distribution and the community structure of the macroinfauna associated to sediments containing razor clams (Tagelus dombeii), during February and September, 2004 on the intertidal flat of Pelluco, Southern Chile. Sediment and macroinfauna samples were collected from sites with and without bivalves, using plastic cylinders divided into 5 equal parts. During both sampling months, the total number of species and the total abundance and biomass of the macroinfauna reached, in almost all sedimentary layers, higher values in the site with $T$. dombeii. In both sampling months, the faunistic composition was significantly different between sites with and without $T$. dombeii. In addition, the similarity in the faunistic composition between sedimentary layers was higher in the site with $T$. dombeii. The results of this study suggest that the bioturbation process of $T$. dombeii affects the vertical distribution of the associated macroinfauna and that this effect would not be dependent on the time year.
\end{abstract}

Key words: Tagelus dombeii, community structure, bioturbation

\begin{abstract}
Resumen.- La estructura comunitaria de la macroinfauna que habita en planicies intermareales, así como su distribución en la columna de sedimento, puede ser influida por una combinación de factores físicos y biológicos. El objetivo de este estudio fue determinar y comparar la distribución vertical y estructura comunitaria de la macroinfauna asociada a sedimentos con navajuelas (Tagelus dombeii) en la planicie intermareal de Pelluco, sur de Chile, durante febrero y septiembre de 2004. Se recolectaron muestras de sedimento y macroinfauna en sitios con y sin bivalvos, con un cilindro plástico dividido en 5 partes iguales. Durante ambos meses de muestreo, el número de especies, abundancia y biomasa total de la macroinfauna alcanzó, en casi todas las láminas sedimentarias, valores más altos en el sitio con T. dombeii. En ambos meses de muestreo, la composición faunística fue significativamente distinta entre sitios con y $\sin T$. dombeii. Además, la similitud en la composición faunística entre láminas sedimentarias fue mayor en el sitio con $T$. dombeii durante ambos meses de muestreo. Estos resultados sugieren que el proceso de bioperturbación de $T$. dombeii afecta la distribución vertical de la fauna asociada y que este efecto no sería dependiente de la época del año.
\end{abstract}

Palabras clave: Tagelus dombeii, estructura comunitaria, bioperturbación

\section{INTRODUCCIÓN}

Las planicies sedimentarias intermareales son consideradas ecosistemas muy productivos, albergando una rica diversidad de invertebrados bentónicos, generalmente con altas abundancias poblacionales (Dittmann \& Vargas 2001). Esta macroinfauna está compuesta principalmente por poliquetos, oligoquetos, moluscos y crustáceos, entre otros
(Featherstone \& Risk 1977, Jaramillo et al.1984, Richter 1985, Quijón et al. 1996). Estos animales representan una importante fuente de alimento para depredadores tales como aves (Sewell 1996, Botto et al. 1998, Van der Meer et al. 2001), peces y crustáceos decápodos (Quinn 1992, Shaw \& Jenkins 1992, Coull et al. 1995). 
La distribución vertical de la macroinfauna dentro del sedimento en planicies intermareales, puede estar significativamente influenciada por las características del mismo (e.g., Featherstone \& Risk 1977, Quijón 1989). Por ejemplo, el grado de compactación, el cual aumenta con la profundidad (Meadows \& Tait 1989), determina la capacidad de enterramiento de los animales, lo que se relaciona con una mayor abundancia y diversidad de especies registradas en las láminas superiores del sedimento (Reise 1985, Quijón 1989, Quijón \& Jaramillo 1996, Magnusson et al. 2003, Mucha et al. 2004). Otros factores como el modo de alimentación, desplazamiento y tamaño de los organismos también han mostrado influir sobre la profundidad hasta la cual la macroinfauna coloniza el sedimento (Reise 1985). Por lo tanto, la distribución vertical de la macroinfauna depende de la combinación entre factores bióticos y abióticos.

Por otro lado, las características sedimentológicas pueden ser modificadas por la actividad de la macroinfauna, generando cambios significativos en su estructura (e.g., Reise 1985, Hall 1994, Cadée 2001). Diferentes mecanismos de desplazamiento y de alimentación de algunos grupos de la macroinfauna (e.g., bivalvos, poliquetos excavadores y sedimentívoros), tienden a modificar la estructura del sedimento cuando estos organismos ocurren en altas densidades (Rhoads 1974, Rhoads \& Boyer 1982). Por ejemplo, la ingestión de particulas finas y la posterior depositación de las mismas como agregados fecales, aumentan la porosidad del sustrato, disminuye la compactación y facilita la resuspensión de partículas (Rhoads 1974, Reise 1985, Hall 1994). Esta modificación del sedimento ha resultado en que otras especies colonicen niveles más profundos del sedimento (Rhoads et al. 1985, Mortimer et al. 1999, Cadée 2001). Es por esto que Jones et al. (1994) se han referido a estos animales como 'ingenieros de ecosistemas'. Existen otros factores biológicos que generan bioperturbación sobre los fondos sedimentarios, como la depredación (Hall et al. 1994), competencia por espacio (Woodin 1981) y construcción de tubos y galerías (Widdows et al. 1998), que al actuar en combinación también influyen sobre la distribución vertical de la macroinfauna, por ejemplo, induciendo su escape o desplazándola a otros sectores.

Uno de los grupos más característicos de la macroinfauna en planicies intermareales son los bivalvos, los cuales pueden llegar a ocupar grandes extensiones y alcanzar altas densidades poblacionales (Commito \&
Dankers 2001). La actividad biológica de estos organismos ha mostrado producir cambios en el ambiente, tal como incremento en los niveles de sedimento fino y materia orgánica (Crooks 1998, Dame et al. 2001, Duarte et al. 2006) afectando a la pequeña macroinfauna que vive asociada a ellos (poliquetos, oligoquetos, anfípodos) (e.g., Duarte et al. 2006, Jaramillo et al. 2007, Buschbaum et al. 2009). Durante el proceso de filtración, estos animales pueden también afectar el asentamiento de organismos de desarrollo indirecto a través de la ingestión de sus larvas (Woodin 1976, Dittman 1990, Lehane \& Davenport 2002).

Las planicies intermareales son ambientes típicos en la costa interior de los canales nor-patagónicos chilenos (ca. $40-42^{\circ} \mathrm{S}$ ). Densos bancos de mitílidos (e.g., Mytilus edulis chilensis (Hupe, 1854)) y bivalvos infaunales [e.g., Mulinia edulis (King \& Broderip, 1832)] son organismos característicos de estos ambientes (Lardies et al. 2001, Duarte et al. 2006, Jaramillo et al. 2007). Los escasos estudios en estas planicies han estado enfocados a entender el efecto de los bivalvos sobre las características sedimentarias y sobre la macroinfauna asociada (e.g., Jaramillo et al. 1992, Duarte et al. 2006, Jaramillo et al. 2007). Sin embargo, no existen hasta la fecha estudios que evalúen el efecto de los bivalvos infaunales sobre la distribución vertical de los sedimentos y la macroinfauna. Por lo tanto, el objetivo del presente estudio fue comparar la distribución vertical de la macroinfauna y de los sedimentos entre áreas con presencia y ausencia del bivalvo Tagelus dombeii (Sowerby, 1835). Este bivalvo forma densas agrupaciones en las planicies del sur de Chile (Jaramillo et al. 2007) y puede alcanzar aproximadamente una profundidad de enterramiento de $30 \mathrm{~cm}$ (Lardies et al. 2001). A fin de evaluar la consistencia temporal de la distribución de la macroinfauna, se realizó el análisis durante los meses de febrero y septiembre de 2004, correspondiente a la época de verano e invierno, respectivamente.

\section{MATERIALES Y MÉTODOS}

\section{ÁREA DE ESTUDIO}

El sitio de estudio se ubica en la planicie intermareal de

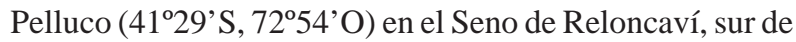
Chile (Fig. 1). La superficie de esta planicie es de $\approx 23 \mathrm{~km}^{2}$. Los rangos mareales durante mareas de sicigia, son $\approx 6 \mathrm{~m}$ (Viviani 1979). 
Figura I. Localización general del área de estudio en la costa interior de los canales Nor-patagónicos chilenos / General location of the study area in the inland coast of the Chilean Nor-patagonic channels

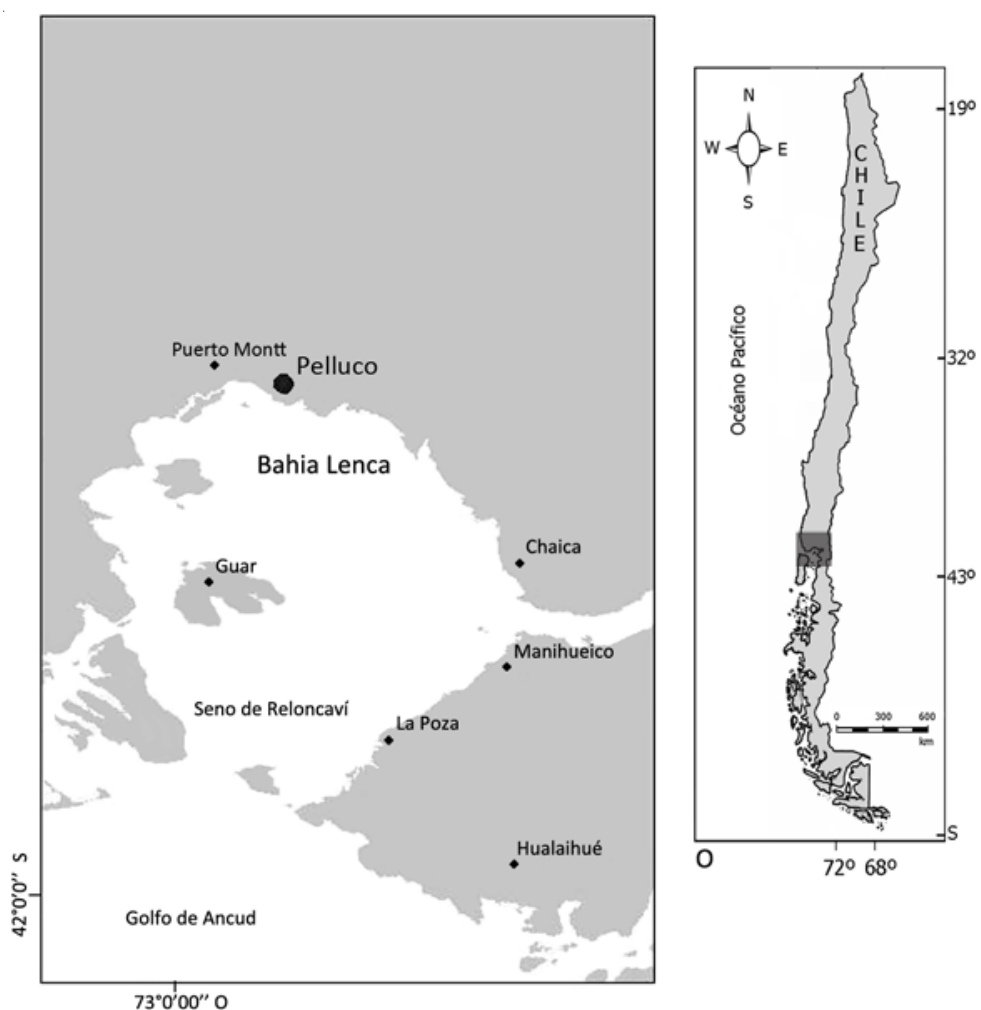

Tratamiento DE LAS MUESTRAS

Los análisis texturales se realizaron siguiendo la metodología de tamizado en húmedo (Anderson et al. 1981), la cual permite separar las fracciones grava (partículas $>2000 \mu \mathrm{m}$ ), arena (partículas entre 63 y 2000 $\mu \mathrm{m}$ ), fango (partículas $<63 \mu \mathrm{m}$ ) y agregados biogénicos representados primariamente por agregados fecales y restos de tubos de poliquetos. Posteriormente, estas fracciones se secaron $\left(60^{\circ} \mathrm{C}\right.$ por $\left.48 \mathrm{~h}\right)$ e incineraron $\left(550^{\circ} \mathrm{C}\right.$ por 4 h). A partir de cálculos de diferencia de peso, se determinaron los porcentajes de materia orgánica (materia orgánica carbonosa o combustible) para cada una de las fracciones y réplicas. Las muestras de fauna fueron analizadas bajo lupa binocular para el conteo e identificación de los organismos hasta el nivel taxonómico más bajo posible. Posteriormente, los organismos se secaron $\left(80^{\circ} \mathrm{C}\right.$ por $\left.72 \mathrm{~h}\right)$ y calcinaron $\left(550^{\circ} \mathrm{C}\right.$ por $\left.4 \mathrm{~h}\right)$, para llevar a cabo estimaciones de biomasa (i.e., peso seco libre de cenizas).

\section{Análisis de los datos}

Para evaluar diferencias en las características sedimentológicas y faunísticas (i.e., número de taxa, fijó en formalina al 10\% diluida en agua de mar. 
abundancia y biomasa de la macroinfauna), entre láminas (i.e., 0-3 cm vs. 3-6 cm) del mismo sitio y entre láminas similares $(0-3 \mathrm{~cm})$ entre sitios, se realizaron análisis de varianza de una vía (Zar 1999). Los supuestos de normalidad y homogeneidad de varianzas fueron evaluados mediante las pruebas estadísticas de Kolmogorov-Smirnov y Bartlett, respectivamente (Zar 1999). Los datos sedimentológicos que no cumplieron con esos supuestos fueron transformados mediante arco seno de la raíz cuadrada de los datos (Zar 1999). En tanto los datos faunísticos que no cumplieron con esos supuestos fueron transformados con $\log (\mathrm{x}+1)$ o raíz cuadrada $(x+0,5)$ (Zar 1999). En los análisis donde se registraron diferencias significativas entre los grupos analizados, se aplicó la prueba a posteriori Tukey's 'HSD' ('Honestly Significant Difference'), con el objetivo de identificar la o las entidades con características significativamente diferentes (Zar 1999). Las variables cuyos valores no fueron posibles de normalizar u homogenizar sus varianzas, se evaluaron mediante la prueba no paramétrica de Kruskal-Wallis (Zar 1999). Todos los datos fueron analizados utilizando el programa STATGRAPHICS Plus 2,0.

La similitud en la estructura comunitaria de la macroinfauna entre sitios y láminas sedimentarias de cada planicie, se evaluó mediante análisis multivariado de escalamiento no métrico multidimensional (EMDNM, Kruskal \& Wish 1978). Para determinar si la estructura comunitaria entre láminas y entre sitios (para una misma lámina) fue significativamente diferente entre sí se realizó un Análisis de Similitudes (ANOSIM, Analysis of Similarities) y conjuntamente con este análisis, se evaluó el porcentaje de disimilitud (SIMPER, Similarity Percentages-species Contribution) entre los distintos sitios de muestreo (Clarke 1993). Los análisis multivariados se basaron en una matriz de similitud calculada mediante el coeficiente de Bray-Curtis, previa transformación de los datos mediante doble raíz cuadrada. Estos análisis se realizaron con el programa PRIMER (Plymouth Routines in Multivariate Ecological Research) (Carr 1997).

\section{Resultados}

\section{SEDIMENTos}

Durante ambos meses de muestreo (febrero y septiembre), el contenido de grava varió entre 3-7\% en el sitio sin bivalvos y entre $5-12 \%$ en el sitio con bivalvos. Los porcentajes de la fracción arena fueron similares en ambos sitios y fechas de muestreo (85-96\%). El sitio con bivalvos registró mayor contenido de fango (1,6-2,4\%) que el sitio sin bivalvos (0,6-1,4\%) durante ambas fechas de muestreo. Durante ambos meses de muestreo, el contenido de agregados biogénicos fue inferior al $1 \%$ en los sitios con y sin bivalvos. El contenido de materia orgánica registró valores cercanos al $1 \%$ en ambos sitios, y fechas de muestreo (Fig. 2).
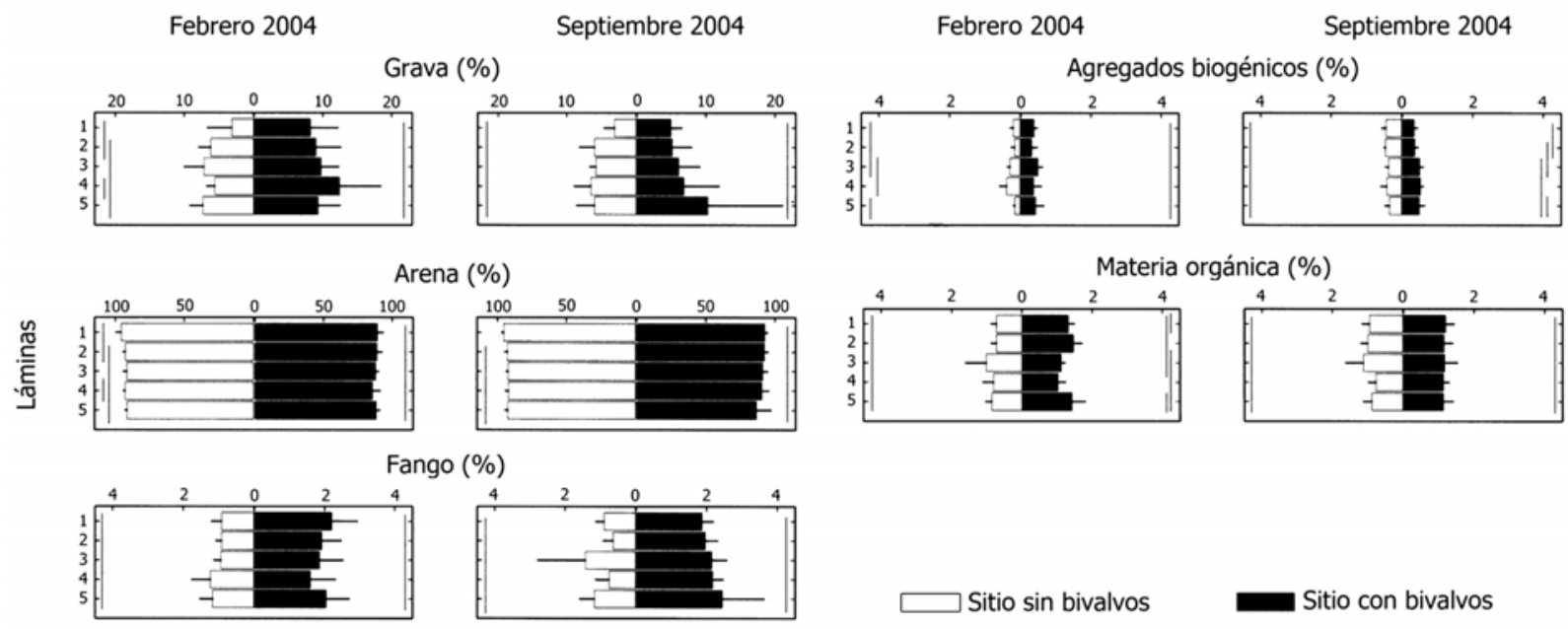

Figura 2. Distribución vertical de los porcentajes de las fracciones grava, arena, fango, agregados biogénicos y contenido de materia orgánica total de los sedimentos de la planicie de Pelluco. Las líneas verticales (a los costados de cada figura) unen láminas que no diferían significativamente entre sí $(\boldsymbol{P}>\mathbf{0 , 0 5}$, resultados de la prueba a posteriori de Tukey) / Vertical distribution of the percentages of gravel, sand, mud, biogenic aggregates and total organic matter of the sediments from the tidal flat of Pelluco. Vertical lines (to each side inside figures) link layers that were not significantly different between one and other $(P>0.05$, results of the a posteriori Tukey test) 
El contenido de grava en el sitio sin bivalvos aumentó gradualmente con la profundidad durante febrero $\left(\mathrm{F}_{(4,20)}\right.$ $=3,10, P<0,05)$, mientras que durante septiembre no se registraron diferencias significativas $\left(\mathrm{F}_{(4,20)}=2,86, P>\right.$ 0,05 ) entre láminas (Fig. 2). Durante ambos meses, ese contenido no presentó diferencias significativas entre láminas sedimentarias en el sitio con bivalvos $\left(\mathrm{F}_{(4,20)}=\right.$ 0,66, $P>0,05$ y $_{(4,20)}=0,36, P>0,05$, para febrero y septiembre, respectivamente, Fig. 2). En el sitio sin bivalvos, el contenido de arena difirió significativamente entre láminas durante febrero $\left(\mathrm{F}_{(4,20)}=3,28, P<0,05\right)$, pero sin mostrar un patrón definido, a la vez que en septiembre, el contenido de arena fue significativamente más alto $\left(\mathrm{F}_{(4,20)}=3,04, P<0,05\right)$ en la lámina superficial (Fig. 2). En el sitio con bivalvos y durante ambos meses, no se registraron diferencias significativas en los porcentajes de arena $\left(\mathrm{F}_{4,20}=0,56, P>0,05 \mathrm{y} \mathrm{F}_{4,20}=0,44, P>0,05\right.$, para febrero y septiembre, respectivamente) entre láminas (Fig. 2). Durante ambos meses de muestreo, el contenido de fango no registró diferencias significativas entre láminas sedimentarias en ambos sitios $\left(\mathrm{F}_{(4,20)}=0,88, P>0,05\right.$ y F$_{(4,}$ ${ }_{20)}=1,05, P>0,05$, para febrero y septiembre en el sitio sin bivalvos, respectivamente; $\mathrm{F}_{(4,20)}=0,56, P>0,05$ y F$_{(4,20)}$ $=0,35, P>0,05$, para febrero y septiembre en el sitio con bivalvos, respectivamente, Fig. 2). Los agregados biogénicos del sitio sin bivalvos registraron diferencias significativas $\left(\mathrm{F}_{(4,20)}=5,47, P<0,05\right)$ entre láminas durante febrero, pero no durante septiembre $\left(\mathrm{F}_{(4,20)}=1,41, P>\right.$ 0,05, Fig. 2). Inversamente, el sitio con bivalvos registró diferencias significativas sólo durante septiembre, donde los contenidos de agregados biogénicos fueron más altos en las láminas más profundas $\left(\mathrm{F}_{(4,20)}=0,94, P>0,05 \mathrm{y} \mathrm{F}_{(4 \text {, }}\right.$ ${ }_{20)}=4,41, P<0,05$ para febrero y septiembre, respectivamente, Fig. 2). El contenido de materia orgánica no presentó diferencias significativas entre láminas sedimentarias durante ambos meses de muestreo en el sitio sin bivalvos $\left(\mathrm{F}_{(4,20)}=0,44, P>0,05 \mathrm{y} \mathrm{F}_{(4,20)}=0,25, P\right.$ $>0,05$ para febrero y septiembre, respectivamente, Fig. 2). En el sitio con bivalvos se registraron diferencias significativas en el contenido de materia orgánica entre láminas solo durante febrero $\left(\mathrm{F}_{(4,20)}=3,92, P<0,05 \mathrm{y} \mathrm{F}_{(4,}\right.$ ${ }_{20)}=0,28, P>0,05$ para febrero y septiembre, respectivamente, Fig. 2).

Las comparaciones entre las características texturales del sedimento entre sitios con y sin bivalvos para cada lámina sedimentaria se muestran en la Tabla 1. Se registraron diferencias significativas en el contenido de grava sólo durante febrero; la cuarta lámina del sitio sin bivalvos registró valores significativamente más bajos que el sitio con bivalvos (Tabla 1, Fig. 2). Durante febrero, el contenido de arena fue, significativamente más alto en el sitio sin bivalvos, con excepción de la segunda y quinta lámina donde no se registraron diferencias significativas;

Tabla 1. Resultado del análisis de ANDEVA de una vía (F: estadígrafo y $P$ : probabilidad estadística) que compara entre sitios con y sin bivalvos, los porcentajes de grava, arena, fango, agregados biogénicos y materia orgánica total, de cada lámina sedimentaria en la planicie de Pelluco. Valores estadísticamente significativos en negrita $(P<0,05) /$ One-way ANOVA results ( $F$ : stadigraph, $P$ : statistic probability) to compare between with and without bivalves, the percentages of gravel, sand, mud, biogenic aggregates and total organic matter, in sedimentary layers at the tidal of Pelluco. Statistical significant values are in bold $(P<0.05)$

\begin{tabular}{|c|c|c|c|c|c|c|c|c|c|c|}
\hline \multirow{2}{*}{ Febrero 2004} & \multicolumn{2}{|c|}{ Grava } & \multicolumn{2}{|c|}{ Arena } & \multicolumn{2}{|c|}{ Fango } & \multicolumn{2}{|c|}{$\begin{array}{l}\text { Agregados } \\
\text { biogénicos }\end{array}$} & \multicolumn{2}{|c|}{$\begin{array}{c}\text { Materia } \\
\text { orgánica }\end{array}$} \\
\hline & & & & & & & & & & \\
\hline Láminas & $\mathrm{F}_{(4,20)}$ & $P$ & $F_{(4,20)}$ & $P$ & $F_{(4,20)}$ & $P$ & $\mathrm{~F}_{(4,20)}$ & $P$ & $\mathrm{~F}_{(4,20)}$ & $P$ \\
\hline $1(0-3 \mathrm{~cm})$ & 5,12 & 0,053 & 7,16 & 0,028 & 15,77 & 0,004 & 11,05 & 0,011 & 31,56 & 0,001 \\
\hline $2(3-6 \mathrm{~cm})$ & 2,43 & 0,157 & 4,74 & 0,064 & 18,27 & 0,003 & 3,41 & 0,102 & 36,50 & $<0,001$ \\
\hline $3(6-9 \mathrm{~cm})$ & 2,36 & 0,162 & 5,79 & 0,042 & 9,62 & 0,015 & 9,40 & 0,016 & 2,45 & 0,117 \\
\hline $4(9-12 \mathrm{~cm})$ & 6,81 & 0,009 & 10,39 & 0,012 & 0,78 & 0,402 & 0,00 & 0,968 & 1,9 & 0,205 \\
\hline $5(12-15 \mathrm{~cm})$ & 1,26 & 0,294 & 4,72 & 0,061 & 6,83 & 0,031 & 8,81 & 0,018 & 10,02 & 0,013 \\
\hline \multicolumn{11}{|l|}{ Septiembre 2004} \\
\hline Láminas & $\mathrm{F}$ & $P$ & $\mathrm{~F}$ & $P$ & $\mathrm{~F}$ & $P$ & $\mathrm{~F}$ & $P$ & $\mathrm{~F}$ & $P$ \\
\hline $1(0-3 \mathrm{~cm})$ & 3,35 & 0,105 & 5,69 & 0,044 & 29,67 & 0,001 & 2,3 & 0,168 & 2,86 & 0,129 \\
\hline $2(3-6 \mathrm{~cm})$ & 0,37 & 0,558 & 0,04 & 0,844 & 33,85 & $<0,001$ & 3,82 & 0,086 & 1,15 & 0,315 \\
\hline $3(6-9 \mathrm{~cm})$ & 0,01 & 0,917 & 0,31 & 0,595 & 2,45 & 0,117 & 4,36 & 0,070 & 0,08 & 0,779 \\
\hline $4(9-12 \mathrm{~cm})$ & 0,01 & 0,909 & 0,41 & 0,538 & 27,76 & 0,001 & 1,55 & 0,249 & 9,43 & 0,015 \\
\hline $5(12-15 \mathrm{~cm})$ & 0,27 & 0,602 & 1,84 & 0,175 & 6,45 & 0,035 & 3,25 & 0,109 & 2,34 & 0,165 \\
\hline
\end{tabular}


en tanto que durante septiembre, la primera lámina registró porcentajes de arena significativamente más altos en el sitio sin bivalvos, mientras que en las demás láminas las diferencias no fueron significativas (Tabla 1, Fig. 2). Durante ambos meses de muestreo, el contenido de fango registró valores significativamente más altos en la mayoría de las láminas del sitio con bivalvos (Tabla 1, Fig. 2). Los porcentajes de agregados biogénicos fueron significativamente diferentes sólo durante febrero, registrándose los valores más altos en las láminas 1 , 3 y 5 del sitio con bivalvos (Tabla 1, Fig. 2). El contenido de materia orgánica fue significativamente más alto en las láminas 1, 2 y 5 del sitio con bivalvos durante febrero y en la cuarta lámina del mismo sitio durante septiembre (Tabla 1, Fig.2).

\section{Macroinfauna}

Durante los muestreos de febrero y septiembre y en ambos sitios, la macroinfauna estuvo numéricamente dominada por anélidos poliquetos, moluscos bivalvos y crustáceos peracáridos (Tabla 2). El sitio ocupado por Tagelus dombeii presentó el mayor número de taxa, especialmente durante febrero (i.e., 40 y 22 durante febrero y septiembre, respectivamente) (Tabla 2).

Durante ambos meses de muestreo en el sitio con bivalvos el número total de especies y abundancia total presentaron la misma tendencia de distribución vertical: valores más altos en la lámina superficial descendiendo
Tabla 2. Número de especies de poliquetos, oligoquetos, gastrópodos, bivalvos y crustáceos peracáridos en la planicie de Pelluco / Number of the species of polychaetes, oligochaetes, gastropods, bivalves and peracarid crustaceans at the tidal flat of Pelluco

\begin{tabular}{|c|c|c|c|c|}
\hline & \multicolumn{2}{|c|}{ Febrero 2004} & \multicolumn{2}{|c|}{ Septiembre 2004} \\
\hline & Sin bivalvos & Con bivalvos & Sin bivalvos & Con bivalvos \\
\hline Polychaeta & 7 & 20 & 3 & 10 \\
\hline Oligochaeta & 3 & 2 & 1 & 1 \\
\hline Gastropoda & 1 & 1 & 1 & 1 \\
\hline Bivalvia & 4 & 6 & 5 & 5 \\
\hline Peracarida & 5 & 7 & 2 & 2 \\
\hline Otros & 1 & 4 & 2 & 3 \\
\hline Total & 21 & 40 & 14 & 22 \\
\hline
\end{tabular}

hacia las láminas inferiores, mientras que en el sitio sin bivalvos la distribución fue en general homogénea a lo largo de la columna del sedimento (Fig. 3). La biomasa fue alta en la última lámina del sitio con bivalvos (300 mg aproximadamente) durante febrero y en la tercera lámina del sitio sin bivalvos (ca., $100 \mathrm{mg}$ ) durante septiembre del mismo año (Fig. 3). La especie más abundante en el sitio sin bivalvos durante febrero fue el anélido Polygordius sp., mientras que en el sitio con bivalvos las especies Polygordius sp. y el anfípodo Corophium sp. fueron las más abundantes. Durante septiembre, la especie dominante en el sitio sin bivalvos fue el gastrópodo Caecum chilense y en el sitio con bivalvos dominaron el
Febrero 2004

Septiembre 2004

Número de especies
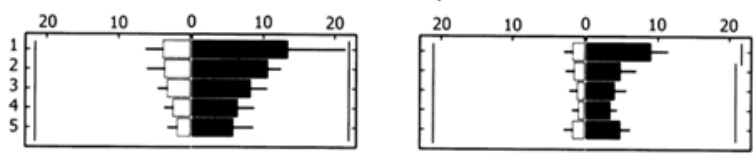

Abundancia total (ind. 0,004 $\mathrm{m}^{-2}$ )
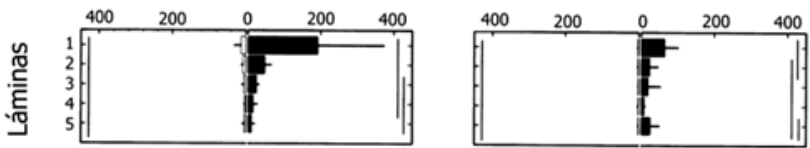

Biomasa total (mg. 0,004 $\mathrm{m}^{-2}$ )
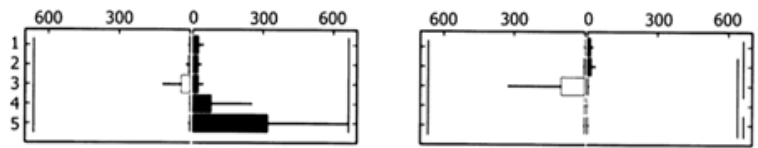

Febrero 2004

Septiembre 2004

Polygordius sp. (ind. 0,004 $\mathrm{m}^{-2}$ )
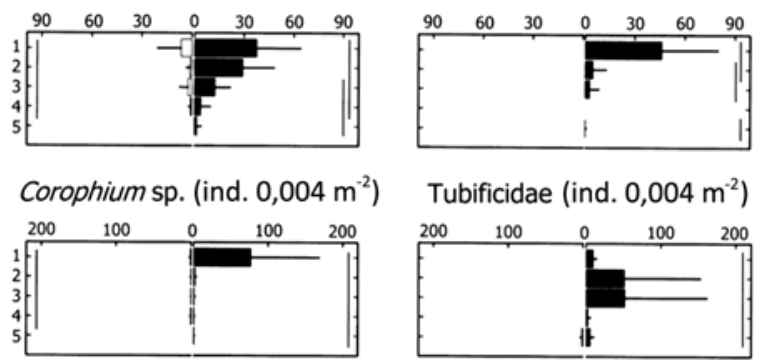

Sitio sin bivalvos
Sitio con bivalvos

Figura 3. Distribución vertical del número de especies, abundancia y biomasa total de la macroinfauna y abundancia de las dos especies más abundantes en la planicie de Pelluco. Las líneas verticales (a los costados de cada figura) unen láminas que no difieren significativamente entre sí $(P>0,05$, resultados de la prueba a posteriori de Tukey) / Vertical distribution of the number of species, total abundance and biomass of the macroinfauna and abundance of the most important species at the tidal flat of Pelluco. Vertical lines (to each side inside figures) link layers that were not significantly different among each others $(P>0.05$, results of the a posteriori Tukey test) 
poliqueto Polygordius sp. y oligoquetos de la familia Tubificidae (Fig. 3).

La distribución vertical del número de especies no presentó diferencias significativas en sitio sin bivalvos durante ambos meses de muestreo $\left(\mathrm{F}_{(4,20)}=1,10, P>0,05\right.$ y $\mathrm{F}_{4,20}=0,69, P>0,05$, para febrero y septiembre, respectivamente, Fig. 3). A su vez, en el sitio con bivalvos, la distribución vertical del número de especies registró diferencias significativas sólo durante septiembre, con valores más altos en la lámina superior $\left(\mathrm{F}_{(4,20)}=2,83, P>\right.$ 0,05 y $_{(4,20)}=7,96, P<0,05$, para febrero y septiembre, respectivamente, Fig. 3). Similar a lo registrado con el número de especies, la distribución vertical de la abundancia total no presentó diferencias significativas en el sitio sin bivalvos durante ambos meses de muestreo $\left(\mathrm{F}_{(4,20)}=2,18, P>0,05\right.$ y $_{(4,20)}=0,61, P>0,05$ para febrero y septiembre, respectivamente, Fig. 3). En el sitio con bivalvos la abundancia total fue, durante ambos meses de muestreo, significativamente mayor en la primera lámina en comparación con las más profundas $\left(\mathrm{F}_{(4,20)}=4,32, P<\right.$ 0,05 y $_{(4,20)}=3,18, P<0,05$ para febrero y septiembre, respectivamente, Fig. 3). Los valores de biomasa total no presentaron diferencias significativas en el sitio sin bivalvos durante ambos meses de muestreo $\left(\mathrm{F}_{(4,20)}=1,06\right.$, $P>0,05$ y F$_{(4,20)}=0,99, P>0,05$, para febrero y septiembre, respectivamente, Fig. 3); mientras que en el sitio con bivalvos la biomasa presentó diferencias significativas entre láminas sólo durante septiembre, pero sin observarse un patrón de incremento o disminución con la profundidad $\left(\mathrm{F}_{(4,20)}=2,65, P>0,05\right.$ y $_{(4,20)}=4,52, P<$ 0,05 , para febrero y septiembre, respectivamente, Fig. 3 ). Durante febrero, la distribución vertical de Polygordius sp. en el sitio sin bivalvos no presentó diferencias significativas entre láminas sedimentarias, mientras que en el sitio con bivalvos registró valores significativamente más altos en láminas superficiales $\left(\mathrm{F}_{(4,20)}=0,57, P>0,05 \mathrm{y}\right.$ $\mathrm{F}_{(4,20)}=5,19 P<0,05$ para el sitio sin y con bivalvos, respectivamente, Fig. 3). Durante septiembre, este poliqueto registró valores significativamente más altos en el nivel superior $\left(\mathrm{F}_{(4,20)}=7,60 P<0,05\right.$, Fig. 3). La otra especie dominante (Corophium sp.) no presentó diferencias significativas en su distribución vertical en ambos sitios de muestreo durante febrero $\left(\mathrm{F}_{(4,20)}=0,50, P\right.$ $>0,05 \mathrm{y} \mathrm{F}_{(4,20)}=2,50, P>0,05$ para sitio sin y con bivalvos, respectivamente, Fig. 3 ). Los oligoquetos de la familia Tubificidae no registraron diferencias significativas en sus abundancias a lo largo de la columna del sedimento durante septiembre (durante febrero esta especie no fue registrada) $\left(\mathrm{F}_{(4,20)}=0,67, P>0,05\right.$, Fig. 3$)$.

Las comparaciones entre las primeras láminas en ambos sitios no mostraron diferencias significativas para ninguna de las variables estudiadas durante febrero; por el contrario, los valores para esas mismas variables en las segundas láminas fueron significativamente más altos en el sitio con bivalvos (Tabla 3, Fig. 3). Para las siguientes láminas (3, 4 y 5), el número total de especies y la

Tabla 3. Resultado del análisis de ANDEVA de una vía (F: estadígrafo y $P$ : probabilidad estadística) que compara el número de especies, abundancia total y biomasa de cada lámina sedimentaria entre sitios con y sin bivalvos, en la planicie de Pelluco. $P<0,05$ ) / One-way ANOVA results ( $F$ : stadigraph, $P$ : statistic probability) to compare between sites with and without bivalves, the number of species, total abundance and biomass of organisms of each sedimentary layer at the tidal flat of Pelluco. Statistical significant values are in bold $(P<0.05)$

\begin{tabular}{|c|c|c|}
\hline $\mathrm{n}^{\circ}$ de especies & $\begin{array}{l}\text { Abundancia total } \\
\text { (ind } 0,004 \mathrm{~m}^{-2} \text { ) }\end{array}$ & $\begin{array}{c}\text { Biomasa } \\
\left(\mathrm{mg} 0,004 \mathrm{~m}^{-2}\right)\end{array}$ \\
\hline
\end{tabular}

\begin{tabular}{lcccccc}
\hline $\begin{array}{c}\text { Febrero 2004 } \\
\text { Láminas }\end{array}$ & \multicolumn{1}{c}{} & & & & \\
& $\mathrm{F}_{(4,20)}$ & $P$ & $\mathrm{~F}_{(4,20)}$ & $P$ & $\mathrm{~F}_{(4,20)}$ & $P$ \\
$1(0-3 \mathrm{~cm})$ & 1,76 & 0,222 & 2,45 & 0,117 & 1,49 & 0,257 \\
$2(3-6 \mathrm{~cm})$ & 26,92 & $\mathbf{0 , 0 0 1}$ & 6,85 & $\mathbf{0 , 0 0 9}$ & 5,75 & $\mathbf{0 , 0 4 3}$ \\
$3(6-9 \mathrm{~cm})$ & 18,12 & $\mathbf{0 , 0 0 3}$ & 20,25 & $\mathbf{0 , 0 0 2}$ & 2,45 & 0,117 \\
$4(9-12 \mathrm{~cm})$ & 12,12 & $\mathbf{0 , 0 0 8}$ & 9,04 & $\mathbf{0 , 0 1 7}$ & 6,85 & $\mathbf{0 , 0 0 9}$ \\
$5(12-15 \mathrm{~cm})$ & 8,51 & $\mathbf{0 , 0 1 9}$ & 1,84 & 0,175 & 5,46 & $\mathbf{0 , 0 4 8}$ \\
& & & & & & \\
Septiembre 2004 & & & & & & \\
Láminas & & & & & & \\
$1(0-3 \mathrm{~cm})$ & 40,26 & $<\mathbf{0 , 0 0 1}$ & 6,85 & $\mathbf{0 , 0 0 9}$ & 48,55 & $<\mathbf{0 , 0 0 1}$ \\
$2(3-6 \mathrm{~cm})$ & 9,63 & $\mathbf{0 , 0 1 5}$ & 4,75 & 0,061 & 3,18 & 0,112 \\
$3(6-9 \mathrm{~cm})$ & 12,86 & $\mathbf{0 , 0 0 7}$ & 6,76 & $\mathbf{0 , 0 3 2}$ & 0,11 & 0,749 \\
$4(9-12 \mathrm{~cm})$ & 22,53 & $\mathbf{0 , 0 0 2}$ & 18,14 & $\mathbf{0 , 0 0 3}$ & 26,11 & $\mathbf{0 , 0 0 1}$ \\
$5(12-15 \mathrm{~cm})$ & 17,07 & $\mathbf{0 , 0 0 3}$ & 6,85 & $\mathbf{0 , 0 0 9}$ & 3,56 & 0,096 \\
\hline
\end{tabular}


abundancia total o la biomasa fueron significativamente más altos en el sitio con bivalvos (Tabla 3, Fig. 3). Durante septiembre, el número total de especies, abundancia total y biomasa total de la macroinfauna fueron significativamente más altos en el sitio con bivalvos en la primera y la cuarta lámina (Tabla 3, Fig. 3), mientras que para las otras láminas (2, 3 y 5) el número total de especies o la abundancia total fueron significativamente más altos en el sitio con bivalvos (Tabla 3, Fig. 3).

Los gráficos de EMDNM muestran que en ambas fechas de muestreo, la distribución de las muestras de la macroinfauna fueron más dispersas en el sitio sin bivalvos (Fig. 4); esto implica que la composición faunística entre las distintas muestras recolectadas en ese sitio presentó menor similitud entre sí, que en la registrada en el sitio con bivalvos. Los porcentajes de disimilitud entre ambos sitios fueron 87,15 y 81,90 durante febrero y septiembre, respectivamente, según los resultados del análisis SIMPER. La especie que más aporto a la disimilitud durante febrero fue Polygordius sp., mientras que el crustáceo Corophium sp. y oligoquetos de la familia Tubificidae fueron las especies que más aportaron a la disimilitud durante septiembre del 2004. Los resultados de la prueba ANOSIM mostraron diferencias significativas $(P<0,05)$ entre ambos sitios, durante ambas fechas de muestreo.

\section{Discusión}

Los resultados de este estudio muestran que la distribución vertical de la macroinfauna fue diferente en los sedimentos ocupados por los bivalvos Tagelus dombeii en comparación a sedimentos sin bivalvos y que este patrón se mantuvo, en general en ambos meses de muestreo (ver Figs. 3 y 4).

Los resultados encontrados en los sedimentos sin bivalvos (donde la distribución vertical de la macroinfauna fue homogénea a lo largo de la columna del sedimento), no coinciden con otros estudios sobre la distribución vertical de la macroinfauna de fondos sedimentarios, donde se ha mencionado que sobre el $90 \%$ de la macroinfauna ocurre en los primeros centímetros de la columna del sedimento, disminuyendo con la profundidad (e.g., Kraeuter \& Fegley 1994, Quijón \& Jaramillo 1996), una situación que ha sido atribuida a la mayor compactación y por consecuencia al menor contenido de agua y oxigenación del sedimento hacia las láminas más profundas (Jaramillo et al. 2007).

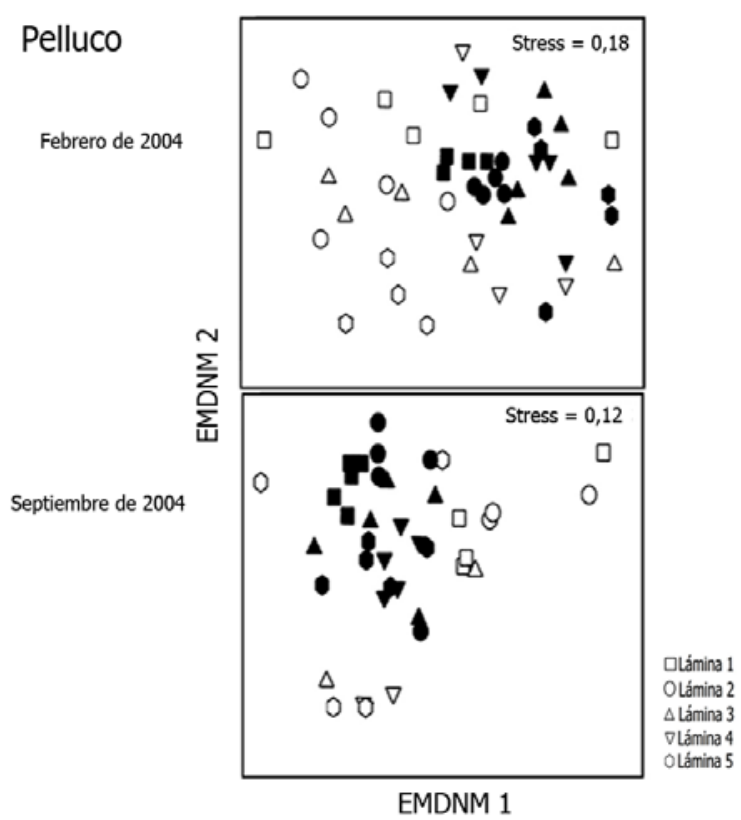

Figura 4. Representación gráfica del análisis EMDNM aplicado a la macroinfauna en la planicie de Pelluco. Los símbolos blancos y los símbolos negros representan las muestras de la macroinfauna recolectada en las láminas sedimentarias de los sitios sin bivalvos y con bivalvos, respectivamente / Graphic display of the NMMDS analysis applied to the macroinfauna from the tidal flat of Pelluco. White and black symbols represent macroinfaunal samples collected from sedimentary layers from sites with and without bivalves, respectively

El proceso de excavación de bivalvos infaunales ha mostrado producir mezcla de partículas de diferentes láminas sedimentarias, disminución de la compactación y una homogenización de la columna sedimentaria (Rhoads \& Young 1971, Nowell et al. 1981, Rhoads et al. 1985, Widdows et al. 1998, Reise 2001, Jaramillo et al. 2007). En este contexto, la gran profundidad de enterramiento que muestra Tagelus dombeii $(20-30 \mathrm{~cm}$, Lardies et al. 2001) podría explicar la distribución más homogénea que registró el contenido de arena y grava en la columna de sedimento en el sitio con bivalvos durante ambos meses de muestreo. Además, la presencia en altas abundancias de $T$. dombeii explicaría en general, los altos valores de fango, agregados biogénicos y materia orgánica en los sitios donde esta especie estuvo presente. Cambios en las características texturales del sedimento como resultado de la presencia de bivalvos es un fenómeno que ha sido previamente reportado en esta y otras planicies (Gutiérrez et al. 2000, Vaughn \& Hakenkamp 2001, Duarte et al. 2006, Jaramillo et al. 2007). 
La macroinfauna asociada a $T$. dombeii mostró un mayor desarrollo hacia láminas más profundas que lo registrado en el sitio en ausencia de este bivalvo. Resultados similares fueron registrados por Bouchet et al. (2009), quienes mostraron que la distribución vertical de los foraminíferos bentónicos en sedimentos intermareales de la costa Atlántica de Francia, estuvo significativamente afectada por la bioirrigación y oxidación del sedimento producida por la macroinfauna, principalmente de bivalvos. Braeckman et al. (2011) registraron, en la costa de Bélgica (Mar del Norte), que el bivalvo Abra alba (Wood 1802) desplazó la comunidad de nemátodos hacia capas más profundas del sedimento, debido a los disturbios físicos que este bivalvo genera en las capas más superficiales y/o a la explotación de recursos alimentarios compartidos. En este estudio no se evaluó el mecanismo específico a través del cual $T$. dombeii influye sobre la distribución vertical de la macroinfauna. Sin embargo, teniendo en cuenta las características texturales de las distintas láminas de sedimento en el sitio con bivalvos, sería posible hipotetizar que la distribución vertical de la macroinfauna podría estar afectada por los cambios sedimentológicos producidos por T. dombeii. Una hipótesis similar fue propuesta por Jaramillo et al. (2007) considerando varias especies de bivalvos en esta misma planicie, aunque en ese estudio no se evaluó la distribución vertical de la macroinfauna.

Los fondos sedimentarios con presencia de Tagelus dombeii, registraron el mayor número de especies y abundancia total de la macroinfauna, en la mayoría de las láminas y durante ambos meses de muestreo. Los estudios realizados hasta la fecha han mostrado que los bivalvos en algunos casos pueden favorecer y en otros casos inhibir o no afectar el desarrollo de la macroinfauna (Reise 1983, Commito 1987, Dittmann 1990, Duarte et al. 2006, Jaramillo et al. 2007). Por ejemplo, los resultados de este estudio son similares a aquellos reportados por Reise (1983) quien registró que agregaciones experimentales del bivalvo Macoma balthica (Linnaeus, 1758) incrementaron la abundancia de la meiofauna en una planicie intermareal del Mar del Norte. Por otro lado, resultados opuestos a los registrados en este estudio fueron encontrados por Black \& Peterson (1988) al comparar sitios sin bivalvos y sitios con las almejas $K$. scalarina (Lamarck, 1818) y Katelysia rhytiphora (Lamy, 1935), en 3 localidades del oeste de Australia, donde la diversidad y densidad de la población de la macroinfauna no difirieron entre sitios. Inicialmente fue propuesto que especies de desarrollo directo serían favorecidas, mientras que aquellas de desarrollo indirecto serían inhibidas en bancos de bivalvos (Woodin 1976, Commito 1987), lo que explicaría los cambios en la composición de especies. Sin embargo, trabajos posteriores han mostrado que el tipo de desarrollo de cada especie, no explicaría estas diferencias (Duarte et al. 2006, Jaramillo et al. 2007). Este estudio también sugiere que el tipo de desarrollo de la macroinfauna no sería el factor determinante para explicar los efectos de T. dombeii, ya que de las 3 especies más abundantes en el sitio con bivalvos 2 presentan desarrollo directo (i.e., Corophium sp. y oligoquetos de la familia Tubificidae) y la otra indirecto (Polygordius sp.). Es importante destacar que no sólo los atributos comunitarios analizados en este estudio difirieron entre sitios con y sin bivalvos, sino también la composición faunística (i.e., composición específica y abundancia relativa). Además, la similitud en la composición faunística entre láminas sedimentarias fue mayor en el sitio con $T$. dombeii, un resultado que reafirma que este bivalvo influye sobre la distribución vertical de la macroinfauna.

Los resultados de este estudio permiten concluir que la distribución vertical de la macroinfauna puede ser significativamente afectada por la presencia de Tagelus dombeii y consecuentemente por otros bivalvos infaunales. Además, en fondos sedimentarios con presencia de $T$. dombeii el número de especies y abundancia total de la macroinfauna fue en general mayor que en sedimentos sin bivalvos. Debido a que T. dombeii y otros bivalvos infaunales que habitan en planicies del sur de Chile son explotadas comercialmente, su extracción podría tener efectos significativos sobre la estructura comunitaria de la macroinfauna asociada y eventualmente sobre todas las especies que ocurren en estos ambientes.

\section{Agradecimientos}

Este estudio fue financiado por CONICYT-CHILE (Proyecto FONDECYT N 1030335 a EJ y Proyecto FONDECYT N 11110407 a CD). Los autores agradecen a Marcia González por su ayuda en la identificación de la fauna. Los comentarios anónimos de los evaluadores de este trabajo, contribuyeron de manera significativa a mejorar el mismo.

\section{LITERATURA CITADA}

Anderson F, L Black, L Mayer \& L Watling. 1981. A temporal and spatial study of a mud flat texture. Northeastern Geology 3: 184-196. 
Black R \& C Peterson. 1988. Absence of preemption and interference competition for space between large suspension-feeding bivalves and smaller infaunal macroinvertebrates. Journal of Experimental Marine Biology and Ecology 120: 183-193.

Botto F, O Iribarne, M Martínez, K Delhey \& M Carrete. 1998. The effect of migratory shorebirds on the benthic species of three Southwestern Atlantic Argentinean Estuaries. Estuaries 21: 700-709.

Bouchet VMP, P-G Sauriau, J-P Debenay, F MermillodBlondin, S Schmidt, J-C Amiard \& B Dupas. 2009. Influence of the mode of macrofauna-mediated bioturbation on the vertical distribution of living benthic foraminifera: First insight from axial tomodensitometry. Journal of Experimental Marine Biology and Ecology 371: 20-33.

Braeckman U, P Provoost, T Moens, K Soetaert, JJ Middelburg, M Vincx \& J Vanaverbeke. 2011. Biological vs. physical mixing effects on benthic food web dynamics. PLoS ONE 6(3): e18078. doi:10.1371/journal.pone.0018078.

Buschbaum C, S Dittmann, J-S Hong, I-S Hwang, M Strasser, M Thiel, N Valdivia, S-P Yoon \& K Reise. 2009. Mytilid mussels: global habitat engineers in coastal sediments. Helgoland Marine Research 63: 47-58.

Cadée G. 2001. Sediment dynamics by bioturbating organisms. In: Reise K (ed). Ecological comparisons of sedimentary shores. Ecological Studies 151: 127-148.

Carr MR. 1997. PRIMER user manual. Plymouth routines in multivariate ecological research, 36 pp. Plymouth Marine Laboratory, Plymouth.

Clarke KR. 1993. Non-parametric multivariate analyses of changes in community structure. Australian Journal of Ecology 18: 117-143.

Commito JA. 1987. Adult-larval interactions: predictions, mussels and cocoons. Estuarine, Coastal and Shelf Science 25: 599-606.

Commito J \& N Dankers. 2001. Dynamics of spatial and temporal complexity in European and North American softbottom mussel beds. In: Reise K (ed). Ecological comparisons of sedimentary shores. Ecological Studies 151: 39-59.

Coull BC, JG Greenwood, DR Fielder \& BA Coull. 1995. Subtropical Australian juvenile fish eat meiofauna: experiments with winter whiting (Sillago maculata) and observations on other species. Marine Ecology Progress Series 125: 13-19.

Crooks J. 1998. Habitat alteration and community-level effects of an exotic mussel, Musculista senhousia. Marine Ecology Progress Series 162: 137-152.

Dame RE, D Bushek \& TC Prins. 2001. Benthic suspension feeders as determinants of ecosystem structure and function in shallow coastal waters. In: Reise K (ed). Ecological comparisons of sedimentary shores. Ecological Studies 151: 11-37.
Dittmann S. 1990. Mussel beds-amensalism or amelioration for intertidal fauna? Helgoländer Meeresuntersuchungen 44: 335-352.

Dittmann S \& J Vargas. 2001. Tropical tidal flat benthos compared between Australia and Central América. En: Reise $\mathrm{K}$ (ed). Ecological comparisons of sedimentary shores. Ecological Studies 151: 127-148.

Duarte C, E Jaramillo, H Contreras \& L Figueroa. 2006. Community structure of the macroinfauna in the sediments below an intertidal mussel bed (Mytilus chilensis (Hupe)) of southern Chile. Revista Chilena de Historia Natural 79: 353-368.

Featherstone RP \& MI Risk. 1977. Effect of tube-building polychaetes on intertidal sediments of the Minas Basin, Bay of Fundy. Journal of Sedimentary Petrology 47: 446-450.

Gutiérrez JL, CG Jones, DL Strayer \& OO Iribane. 2003. Mollusk as ecosystem engineers: the role of shell production in aquatic habitats. Oikos 101: 79-90.

Hall SJ. 1994. Physical disturbance and marine benthic communities: life in unconsolidated sediments. Oceanography and Marine Biology: an Annual Review 32: 179-239.

Hall SJ, D Raffaelli \& SF Thrush. 1994. Patchiness and disturbance in shallow water benthic assemblages. In: Giller PS, AG Hildrew \& D Raffaelli (eds). Aquatic ecology, pp. 333-376. Blackwell Science, Oxford.

Jaramillo E, S Mulsow, M Pino \& H Figueroa. 1984. Subtidal benthic macroinfauna in an estuary of south Chile: distribution pattern in relation to sediment types. Marine Ecology 5: 119-133.

Jaramillo E, C Bertrán \& A Bravo. 1992. Mussel biodeposition in an estuary in southern Chile. Marine Ecology Progress Series 82: 85-94.

Jaramillo E, H Contreras \& C Duarte. 2007. Community structure of the macroinfauna inhabiting tidal flats characterized by the presence of different species of burrowing bivalves in Southern Chile. Hydrobiologia 580: 85-96.

Jones CG, JH Lawton \& M Shachak. 1994. Organism as ecosystem engineers. Oikos 69: 373-386.

Kraeuter J \& S Fegley. 1994. Vertical disturbance of sediment by horseshoe crabs (Limulus polyphemus) during their spawning season. Estuaries 17: 288-294.

Kristensen E. 1988. Benthic fauna and biogeochemical processes in marine sediments: microbial activities and fluxes. En: Blackburn TH \& J Sorensen (eds). Nitrogen cycle in coastal marine enviromnents, pp. 275-299. John Wiley, Chichester.

Kruskal JB \& M Wish. 1978. Multidimensional scaling. Sage University Paper Series on Quantitative Applications in the Social Sciences 07-011. Sage Publications, Newbury Park.

Lardies MA, E Clasing, JM Navarro \& RA Stead. 2001. Effects of environmental variables on burial depth of two infaunal bivalves inhabiting a tidal flat in southern Chile. Journal of the Marine Biological Association of the United Kingdom 81: 809-816. 
Lehane C \& J Davenport. 2002. Ingestion of mesozooplankton by three species of bivalves; Mytilus edulis, Cerastoderma edule and Aequipecten opercularis. Journal of the Marine Biological Association of the United Kingdom 82: 615-619.

Magnusson K, S Agrenius \& R Ekelund. 2003. Distribution of tetrabrominated diphenyl ether and its metabolites in soft-bottom sediment and macrofauna species. Marine Ecology 255: 155-170.

Meadows PS \& J Tait. 1989. Modification of sediment permeability and shear strength by two burrowing invertebrate. Marine Biology 101: 75-82.

Mortimer RJG, JT Davey, MD Krom, PG Watson, PE Frickers \& RJ Clifton. 1999. The effect of macrofauna on porewater profiles and nutrient fluxes in the intertidal zone of the Humber Estuary. Estuarine, Coastal and Shelf Science 48: 683-699.

Mucha AP, MT Vasconcelos \& AA Bórdalo. 2004. Vertical distribution of the macrobenthic community and its relationships to trace metals and natural sediment characteristics in the lower Douro estuary, Portugal. Estuarine, Coastal and Shelf Science 59: 663-673.

Nowell AR, PA Jumars \& JE Eckman. 1981. Effects of biological activity on the entrainment of marine sediments. Marine Geology 42: 133-153.

Quijón P. 1989. Fluctuaciones temporales de la macroinfauna y características sedimentológicas, en el intermareal estuarial del río Queule, centro-sur de Chile. Tesis de Licenciatura en Ciencias, Facultad de Ciencias, Universidad Austral de Chile, Valdivia, 27 pp.

Quijón P \& E Jaramillo. 1996. Seasonal vertical distribution of the intertidal macroinfauna in an estuary of south-central Chile. Estuarine, Coastal and Shelf Science 43: 53-663.

Quijón P, E Jaramillo \& M Pino. 1996. Macroinfaunal assemblages associated with mussel and clam beds in an estuary of southern Chile. Estuaries 19: 62-74.

Quinn RH. 1992. Fisheries resources of the Moretón Bay Región, 52 pp. Queensland Fish Management Authority, Brisbane.

Reise K. 1983. Biotic enrichment of intertidal sediments by experimental aggregates of the deposit-feeding bivalve Macoma balthica. Marine Ecology Progress Series 12: 229236.

Reise K. 1985. Tidal flat ecology: An experimental approach to species interaction, 191 pp. Springer-Verlag, New York.

Reise K. 2001. Synthesis: comparative ecology of sedimentary shores. In: Reise K (ed). Ecological comparisons of sedimentary shores. Ecological Studies 151: 357-371.
Rhoads DC. 1974. Organism-sediment relations on the muddy sea floor. Oceanography and Marine Biology: an Annual Review 12: 263-300.

Rhoads DC \& DK Young. 1971. The influence of depositfeeding organism on sediment stability and community trophic structure. Journal of Marine Research 28: 150-178.

Rhoads DC \& LF Boyer. 1982. The effects of marine benthos on physical properties of sediments: a successional perspective. In: McCall PL \& MJS Tevesz (eds). Animalsediment relations. The biogenic alteration of sediments, pp. 3-52. Plenum Press, New York.

Rhoads DC, DF Boesch, T Zhican, X Fengsahn, H Liqiang \& K Nielsen. 1985. Macrobenthos and sedimentary facies on the Changjiang delta platform and adjacent continental shelf, East China Sea. Continental Shelf Research 4: 189213.

Richter W. 1985. Distribution of the soft-bottom macroinfauna in an estuary of Southern Chile. Marine Biology 86: 93100.

Sewell M. 1996. Detection of the impact of predation by migratory shorebirds: an experimental test in the Fraser River estuary, British Columbia (Canada). Marine Ecology Progress Series 144: 23-40.

Shaw M \& GP Jenkins. 1992. Spatial variation in feeding, prey distribution and food limitation of juvenile flounder Rhombosolea tapirina Gunther. Journal of Experimental Marine Biology and Ecology 165: 1-21.

Van der Meer J, T Piersma \& JJ Beukema. 2001. Population dynamics of benthic species on tidal flats: the possible roles of shorebirds predation. In: Reise K (ed). Ecological comparisons of sedimentary shores. Ecological Studies 151: 317-331.

Vaughn CC \& CC Hakenkamp. 2001. The functional role of burrowing bivalves in freshwater ecosystems. Freshwater Biology 46: 1431-1446.

Viviani CA. 1979. Ecogeografía del litoral chileno. Studies on Neotropical Fauna and Environment 14: 65-123.

Widdows J, M Brinsley, P Salkeld \& M Elliot. 1998. Use of annular flumes to determine the influence of current velocity and material flux at the sediment-water interface. Estuaries 21: 552-559.

Woodin SA. 1976. Adult-larval interactions in dense infaunal assemblages: patterns of abundance. Journal of Marine Research 34: 25-41.

Woodin SA. 1981. Disturbance and community structure in a shallow water sand flat. Ecology 62: 1052-1066.

Zar JH. 1999. Biostatistical analysis, 663 pp. Prentice Hall, Englewood Cliffs.

Recibido el 11 de octubre de 2011 y aceptado el 24 de septiembre de 2012

Editor Asociado: Gabriela Muñoz C. 